\title{
Expression and roles of syndecan-4 in dental epithelial cell differentiation
}

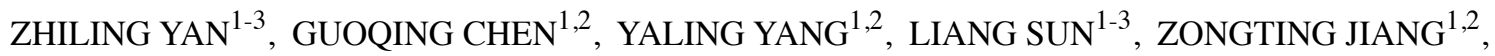 \\ LIAN FENG ${ }^{1,2}$, MEI YU ${ }^{1,2}$, WEIHUA GUO ${ }^{2,4}$ and WEIDONG TIAN ${ }^{1,3}$ \\ ${ }^{1}$ State Key Laboratory of Oral Diseases, ${ }^{2}$ National Engineering Laboratory for Oral Regenerative Medicine, \\ West China Hospital of Stomatology, Sichuan University; Departments of ${ }^{3}$ Oral and Maxillofacial Surgery \\ and ${ }^{4}$ Pedodontics, West China School of Stomatology, Sichuan University, Chengdu, Sichuan 610041, P.R. China
}

Received April 29, 2014; Accepted August 1, 2014

DOI: $10.3892 /$ ijmm.2014.1910

\begin{abstract}
Syndecan-4 (SDC4), a transmembrane heparan sulfate proteoglycan, acts as a signal transducer. It affects the growth and differentiation of a number of tissues and organs. However, the specific mechanisms through which SDC4 regulates the differentiation of dental epithelial cells (amelogenesis) and tooth development remains largely unknown. In the present study, to identify the SDC4-regulated processes in dental epithelial cells, the SDC4 expression pattern was examined in mouse molar and postnatal incisor tooth germs during the late bell stage of development. Small interfering RNA (siRNA) was designed for this study and used to downregulate SDC4 expression in the rat dental epithelial cell line, HAT-7. The results revealed that SDC4 was mainly present in the oral epithelium, the dental epithelial cells of enamel organs in the molars and the cervical loops in the incisors. When the inner enamel epithelial cells gave rise to ameloblasts, however, the loss of SDC4 expression was evident. SDC4 was also expressed in stratum intermedium (SI) cells in the incisors and in dental mesenchymal cells adjacent to the cervical loops in molars (E18) and postnatal incisors. Fibroblast growth factor 10 (FGF10) promoted proliferation and slightly decreased cell differentiation. The knockdown of SDC4 using specific siRNA led to a decrease in cell proliferation and a highly significant increase in amelogenin, ameloblastin, kallikrein 4 and matrix
\end{abstract}

Correspondence to: Dr Weihua Guo, National Engineering Laboratory for Oral Regenerative Medicine, West China Hospital of Stomatology, Sichuan University, No. 14 3rd Section of Ren Min Nan Road, Chengdu, Sichuan 610041, P.R. China

E-mail: guoweihua943019@163.com

Professor Weidong Tian, State Key Laboratory of Oral Diseases, West China Hospital of Stomatology, Sichuan University, No. 14 3rd Section of Ren Min Nan Road, Chengdu, Sichuan 610041, P.R. China E-mail: drtwd@sina.com

Key words: syndecan-4, dental epithelial cell, differentiation, small interfering RNA, tooth development metalloproteinase 20 expression, molecules that are known to participate in the formation of enamel. These effects were attenuated by FGF10, which upregulated SDC4 expression. Taken together, these results suggest that SDC4 participates in amelogenesis, and FGF10 may modulate dental epithelial cell behaviors through the regulation of SDC4 expression.

\section{Introduction}

Syndecan (SDC)4 is a cell surface heparan sulfate proteoglycan (HSPG). Its heparan sulfate (HS) chains and core protein could control the stability, movement and reception of diffusible heparin-binding growth factors (1). In addition, it can form physical connections between the extracellular matrix (ECM) and intracellular signaling to affect the growth and differentiation of a number of tissues and organs $(2,3)$.

SDC4 is highly complex by virtue of its external side chains, and thus interacts with a variety of ligands, such as vascular endothelial growth factors (VEGFs), platelet-derived growth factors (PDGFs) and fibroblast growth factors (FGFs) (4). At the cell membrane, SDC4 stabilizes the interactions between ligands and receptors by forming a ternary complex, which has been demonstrated in several signaling pathways $(5,6)$. Proteolytic cleavage and shedding of its extracellular domain can spread FGF signaling to adjacent cells, thus regulating the local reception of FGF signaling activity (7). Its cytoplasmic domains can also initiate FGF-induced signaling independently of FGFRs through the activation of Rho GTPases, such as Rac1, which plays an essential role in the dental epithelium, involving cell-matrix interactions and matrix biomineralization $(8,9)$.

FGF signaling plays critical roles in tooth development (10). In molar tooth development, FGF4, 8 and 9 act as epithelial signals, mediating inductive interactions between the dental epithelium and the mesenchyme during the initiation of tooth development and the regulation of tooth shape (11). FGF10 signaling is also important for the continuous growth of murine incisors as it maintains dental epithelial stem cells and regulates enamel formation. FGF10 knockout mice lack cervical loop structure and have enamel defects $(12,13)$.

In the present study, to determine the roles of SDC4 in rodent tooth development, particularly in dental epithelial cell differentiation, the expression patterns of SDC4 during the late bell 
stage of development of molar tooth germs and its localization in postnatal mice incisors were examined. The rat dental epithelial cell line, HAT-7, was used to examine the interactions between SDC4 and FGF10 and their effects on dental epithelial cell proliferation and differentiation. Our results reveal the temporospatial expression of SDC4 in molars and incisors during amelogenesis, providing some evidence that FGF10 signaling interacts with SDC4 to affect dental epithelial cell behavior.

\section{Materials and methods}

Animals and tissue preparation. The animal experimental protocol was approved by the Ethics Committee of West China College of Stomatology, Sichuan University, Chengdu, China. C57BL/6J mice were purchased from the experimental Animal Laboratory of Sichuan University. The appearance of a vaginal plug was designated as day 0 . Heads were dissected from embryonic mice on embryonic day 18 (E18) and from newborn mice on postnatal days (P)2, 4 and 7. These heads were fixed in freshly prepared $4 \%$ paraformaldehyde overnight at $4^{\circ} \mathrm{C}$, cut in half along the midline, dehydrated, embedded in paraffin wax, serially sectioned at $6 \mu \mathrm{m}$ and either stained with hematoxylin and eosin (H\&E) or used in immunohistochemistry.

Immunohistochemistry. Tooth germs (incisors and molars), sectioned as described above, were incubated with rabbit polyclonal anti-SDC4 antibody (1:500; Abcam, Cambridge, MA, USA). The sections were then stained using a 3,3'-diaminobenzidine DAB kit (Dako, Carpinteria, CA, USA). Immunohistochemical control was performed by replacing the primary antibody with phosphate-buffered saline (PBS). These immunostained sections contained no specific immunoreactions and were counterstained with hematoxylin. The immune reactions were visualized under a light microscope (Olympus BX43F; JEOL, Tokyo, Japan).

Cell culture and transfection with small interfering RNA (siRNA). HAT-7 cell line was graciously provided by Professor Hidemitsu Harada, Department of Oral Anatomy and Developmental Biology, Osaka University Graduate School of Dentistry, Osaka, Japan. The HAT-7 cells were plated with Dulbecco's modified Eagle's medium/F-12 (DMEM/F-12; Gibco-BRL, Grand Island, NY, USA) supplemented with $10 \%$ fetal bovine serum (FBS) and penicillin-streptomycin. The cells were grown in a humidified atmosphere at $37^{\circ} \mathrm{C}$ with $5 \% \mathrm{CO}_{2}$, and the medium was changed every 3 days. For transient transfections, the cells were cultured in medium without antibiotics $24 \mathrm{~h}$ prior to transfection. Three different nucleotides (SDC4 siRNA 1, 2 and 3) targeting rat SDC4 mRNA (GenBank accession no. NM_012649.2) were designed and tested for silencing. Their sequences are shown in Table I. The cells were transfected with the siRNAs $(50 \mathrm{nM})$ and Lipofectamine ${ }^{\circledR}$ RNAiMAX transfection reagent (Invitrogen, Carlsbad, CA, USA) (1:1 v/v). Non-silencing siRNA with no homology to any known mammalian gene was used as a negative control. The RNAi-mediated knockdown of SDC4 expression was verified by quantitative reverse transcription-polymerase chain reaction (RT-qPCR) and western blot analysis.

Cell proliferation assay. Cells transfected with SDC4 siRNA were used $24 \mathrm{~h}$ after transfection, and recombinant mouse
Table I. Sequences of the 3 different nucleotides (SDC4 siRNA 1 , 2,3 ) targeting rat SDC4 mRNA expression (GenBank accession no. NM_012649.2).

\begin{tabular}{lc}
\hline Nucleotide & \multicolumn{1}{c}{ Sequence $\left(5^{\prime} \rightarrow 3^{\prime}\right)$} \\
\hline siRNA 1 & \\
Sense strand & GGCAGAUACUUCUCUGGAGdTdT \\
Antisense strand & dTdTCCGUCUAUGAAGAGACCUC \\
siRNA 2 & \\
Sense strand & CCUUGGUGCCACUAGAUAAdTdT \\
Antisense strand & dTdTGGAACCACGGUGAUCUAUU \\
siRNA 3 & \\
Sense strand & GGUCUUGGCAGCUCUGAUUdTdT \\
Antisense strand & dTdTCCAGAACCGUCGAGACUAA \\
\hline
\end{tabular}

SDC4, syndecan-4; siRNA, small interfering RNA.

Table II. Oligonucleotide primer sequences utilized in RT-qPCR.

\begin{tabular}{lll}
\hline $\begin{array}{l}\text { Gene } \\
\text { name }\end{array}$ & Primer sequence $\left(5^{\prime} \rightarrow 3^{\prime}\right)$ & $\begin{array}{c}\text { Product } \\
\text { size }(b p)\end{array}$ \\
\hline
\end{tabular}

\begin{tabular}{lll}
\hline SDC1 & F: TTCTCATTGTGGGGAGGTCTA & 82 \\
& R: CTGCTGGGGCTCTAAAACAG &
\end{tabular}

SDC2 F: CACCGAGAAACATTCAGACAA 88

R: GGCAAAGAGAAAGCCAATCA

SDC3 F: TGCGGTTCATTCCTGACATA 98

R: GAGTTCCTCAAACGGGGTATC

SDC4 F: GGGCAAGAAACCCATCTACA 100

$$
\text { R: TGAAGTCCAAGCAGCACTCA }
$$

AMBN F: GAGAAAGGAGAGGGTCCAGAAG 126

R: GTCATTGGGGAAAGCAAGAAGT

AMGN F: ACCTCTGCCTCCACTGTTCTC 102

R: ACTTCTTCCCGCTTGGTCTT

ENAM F: GGTGTCTTCCCTCTCCCTAAA R: AGTGGTTTGCCATTGTCTTTCT

KLK4 F: CCGAACTACAATGACCCTTCTT R: TCAGATGCTACCGAGAGATTCA

MMP20 F: GCCTTGCTGTCCTTGTCAC 95 R: GAGGTGGTAGTTGCTCCTGAAG

RUNX2 F: GAAATGCCTCTGCTGTTATGAA R: CCGTTATGGTCAAAGTGAAACTC

GAPDH F: ATCATCCCTGCCTCTACTGG R: CTGCTTCACCACCTTCTTGA

F, forward; R, reverse; SDC4, syndecan-4; AMBN, ameloblastin; AMGN, amelogenin; ENAM, enamelin; KLK4, kallikrein 4; MMP20, matrix metalloproteinase 20; GAPDH, glyceraldehyde 3-phosphate dehydrogenase.

FGF10 at $10 \mathrm{ng} / \mathrm{ml}$ (R\&D Systems, Minneapolis, MN, USA) was added for a further $48 \mathrm{~h}$. Cell proliferation was assayed 

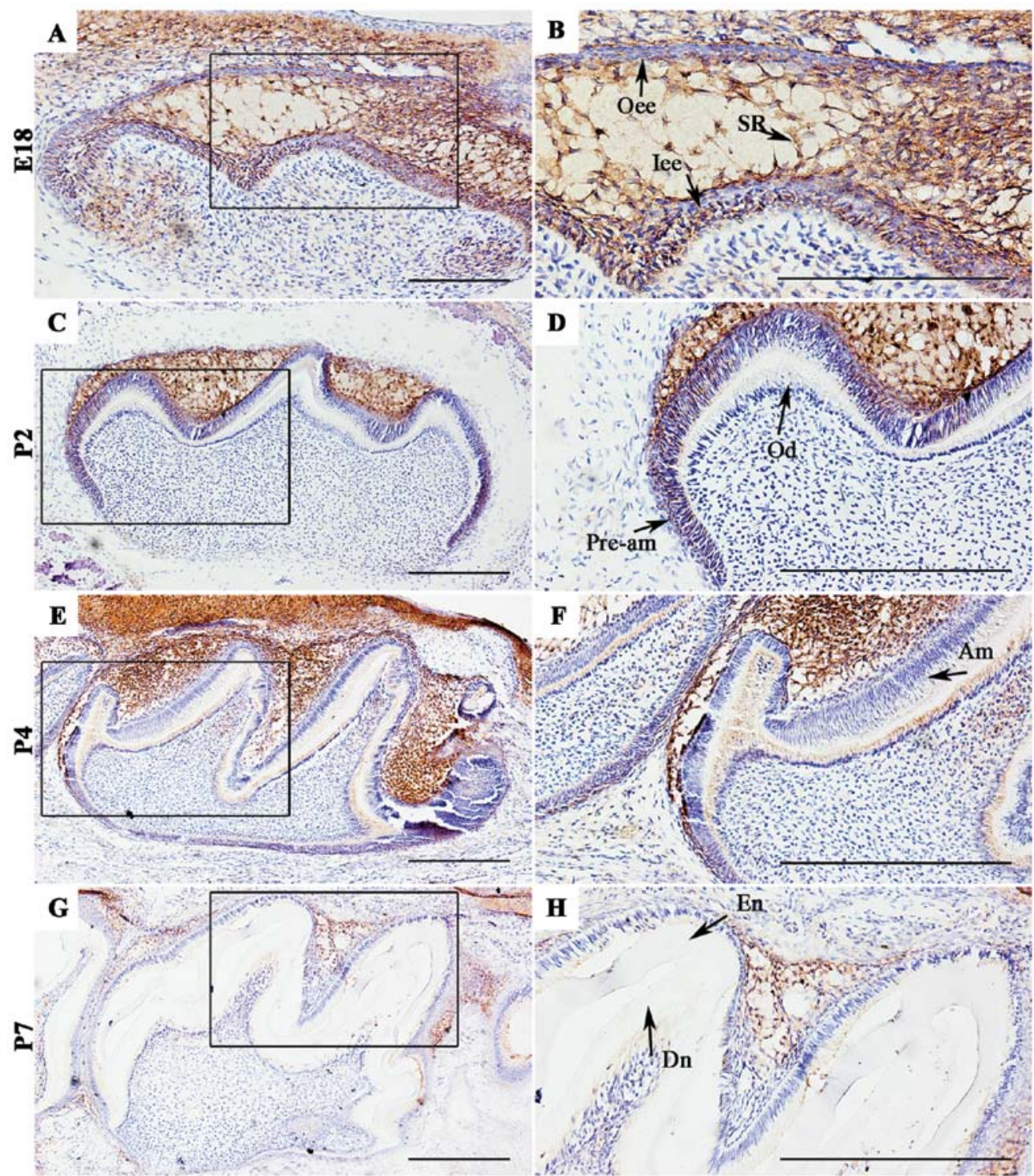

Figure 1. Localization of syndecan-4 (SDC4) in first molar tooth germs of mice at embryonic day 18 (E18) and newborn mice at postnatal day(P)2, P4 and P7. (A and B) At the late bell stage of development (E18), SDC4 was intensely expressed in the oral epithelium, stellate reticulum (SR) cells, and inner and outer enamel epithelium. It was also observed in mesenchymal cells adjacent to the cervical loop structure. (C and D) At P2, SDC4 was observed among the pre-ameloblasts. (D-F) At P4, SDC4 protein was faintly detected in newly differentiated ameloblasts and odontoblasts. (G and H) At P7, SDC4 was almost undetectable in ameloblasts and odontoblasts. The expression of SR was also decreased. (B, D, F and H) Magnifications of the dark boxed areas in (A, C, E and G). Oee, outer enamel epithelium; Iee, inner enamel epithelium; Pre-am, pre-ameloblasts; Od, odontoblasts; Am, ameloblasts; De, dentin; En, enamel. Scale bar, $100 \mu \mathrm{m}$.

with a BrdU incorporation experiment. Briefly, cells on the coverglass were incubated with $\mathrm{BrdU}(10 \mu \mathrm{M}$; Sigma-Aldrich, St. Louis, MO, USA) for $1 \mathrm{~h}$ and fixed by immersing the cells in $4 \%$ paraformaldehyde for $10 \mathrm{~min}$. DNA denaturation was performed in $2 \mathrm{~N} \mathrm{HCI}$ for $30 \mathrm{~min}$ at $37^{\circ} \mathrm{C}$ prior to incubation with $6 \mu \mathrm{g} / \mathrm{ml}$ anti-BrdU antibody (Millipore, Billerica, CA, USA) overnight at $4^{\circ} \mathrm{C}$. Alexa-Fluor 488 -labeled goat antimouse IgGs (1:1,000; Invitrogen) was used as a secondary antibody. After counterstaining with 4',6-diamidino2-phenylindole (DAPI) (1:1,000; Sigma-Aldrich), the number of BrdU-positive cells was quantified under a fluorescence microscope (Leica, Wetzlar, Germany) and the results are presented as a ratio.

$R T-q P C R$. Total RNA was extracted from the cells using TRIzol reagent (Invitrogen). cDNA was prepared from $5 \mu \mathrm{g}$ total RNA using a First Strand cDNA Synthesis kit (Thermo Fisher Scientific, Waltham, MA, USA). After the cells were transfected with SDC4 siRNAs, the expression of SDC4 mRNA was quantified by quantitative PCR using a SYBR-Green probe and used to screen the most effective siRNA. After the cells were transfected with siRNA 1 for $48 \mathrm{~h}$, the expression of SDC1, 2, 3 and 4 was also assayed. Amelogenesis-related gene expression was assessed by RT-qPCR following transfection and/or FGF10 treatment. The target gene primers used are listed in Table II. The genes of interest were normalized to the levels of glyceraldehyde 3-phosphate dehydrogenase (GAPDH) and presented relative to the control levels.

Western blot analysis. Cell lysates were collected from siRNA-and/or FGF10-treated HAT-7 cells to screen the effective siRNA and to evaluate the effects of siRNA and FGF10 on 


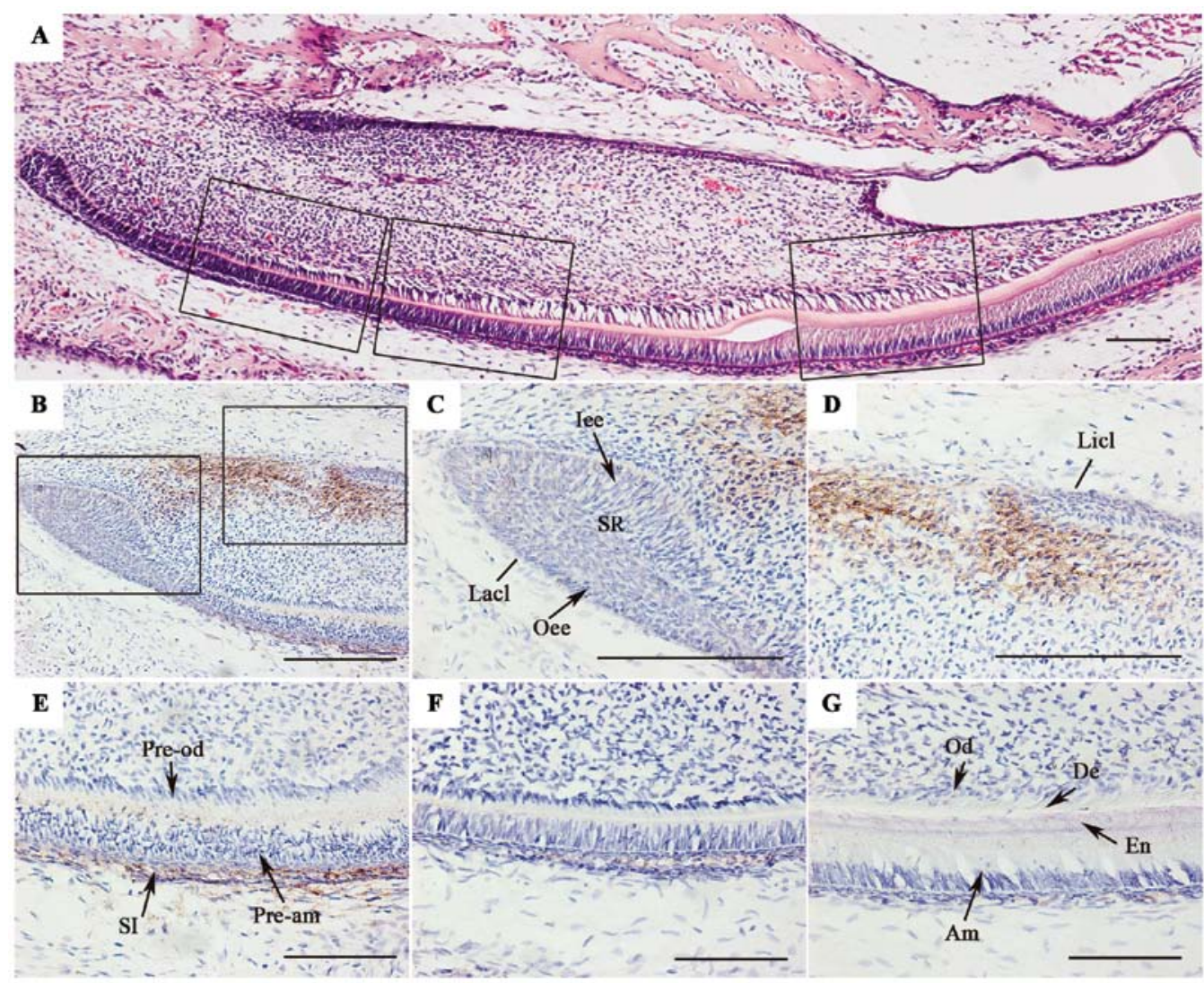

Figure 2. Localization of syndecan-4 (SDC4) in incisors of postnatal mice. (A) Hematoxylin and eosin (H\&E) staining of postnatal mouse incisors. (B-D) SDC4 protein expression was detected in labial and lingual cervical loops and surrounding mesenchymal cells. (E-G) From the proximal to distal parts of the incisor, SDC4 protein expression was not detectable in pre-ameloblasts, pre-odontoblasts, ameloblasts or odontoblasts. It was restricted to stratum intermedium (SI) cells, and the staining in this area gradually declined along with cell differentiation. (C and D) Magnifications of the boxed areas in (B), and (E-G) are magnifications of the dark boxed areas in (A). Lacl, labial cervical loop; Licl, lingual cervical loop; Oee, outer enamel epithelium; SR, stellate reticulum; Iee, inner enamel epithelium; Pre-od, pre-odontoblasts; Pre-am, ameloblasts; Od, odontoblasts; Am, ameloblasts; De, dentin; En, enamel. Scale bar, $100 \mu \mathrm{m}$.

the protein expression of SDC4, amelogenin (AMGN), ameloblastin (AMBN) and kallikrein 4 (KLK4). The samples were boiled in Laemmli sample buffer and loaded onto a sodium dodecyl sulfate-polyacrylamide gel (SDS-PAGE) followed by transfer onto nitrocellulose membranes. The primary antibodies used were rabbit polyclonal anti-SDC4 (1:500; Abcam), anti-AMGN (1:500), anti-AMBN (1:500) and anti-KLK4 (1:500) antibodies (all from Santa Cruz Biotechnology, Inc., Santa Cruz, CA, USA). Anti- $\beta$-actin (1:1,000; Abcam) antibody was used as an internal standard.

Statistical analysis. All experiments were performed independently at least 3 times. All data are presented as the means \pm standard deviation (SD). Statistical significance was assessed using two-tailed Student's t-tests for 2 groups or the analysis of variance Tukey's test for more than 2 groups. Values of $\mathrm{P}<0.05$ and $\mathrm{P}<0.001$ were considered to indicate statistically signficant differences.

\section{Results}

SDC4 expression in mouse molars during the late bell stage of development. SDC4 expression was mainly detected in the oral epithelium and dental epithelial cells, including the inner and outer enamel epithelium and stellate reticulum (SR) cells (Fig. 1B). It was also found in mesenchymal cells adjacent to the lateral sides of the epithelial bud at E18 (Fig. 1A). On P2, SDC4 was expressed in the pre-ameloblasts (Fig. 1C and D), but its expression was almost undetectable in the ameloblasts on P4 and P7 (Fig. 1E-H). Immunostaining in the SR also decreased along with amelogenesis (Fig. 1).

SDC4 expression in mouse postnatal incisors. SDC4 expression was detected in the labial cervical loop, lingual cervical loop and surrounding mesenchymal cells (Fig. 2B-D). From cervical loops to the distal part of incisors, SDC4 protein expression was not detectable in pre-ameloblasts, pre-odontoblasts, ameloblasts or odontoblasts. It was restricted to the stratum intermedium (SI) cells, and the staining in this area gradually declined along with cell differentiation (Fig. 2E-G).

SDC4 silencing in HAT-7 cells. For the analysis of molecular function, SDC4 was knocked down in HAT-7 cells, an immortalized dental epithelial cell line derived from the cervical stem cell population of 6-day-old rat mandibular incisors [provided by Professor Hidemitsu Harada (14); (Fig. 3A)]. RT-qPCR and western blot analysis revealed that SDC4 was successfully knocked down using siRNA. siRNA 1 showed the highest inhibition efficiency and had no effect on the gene expression of SDC1, 2 or 3 (Fig. 3B-D). 

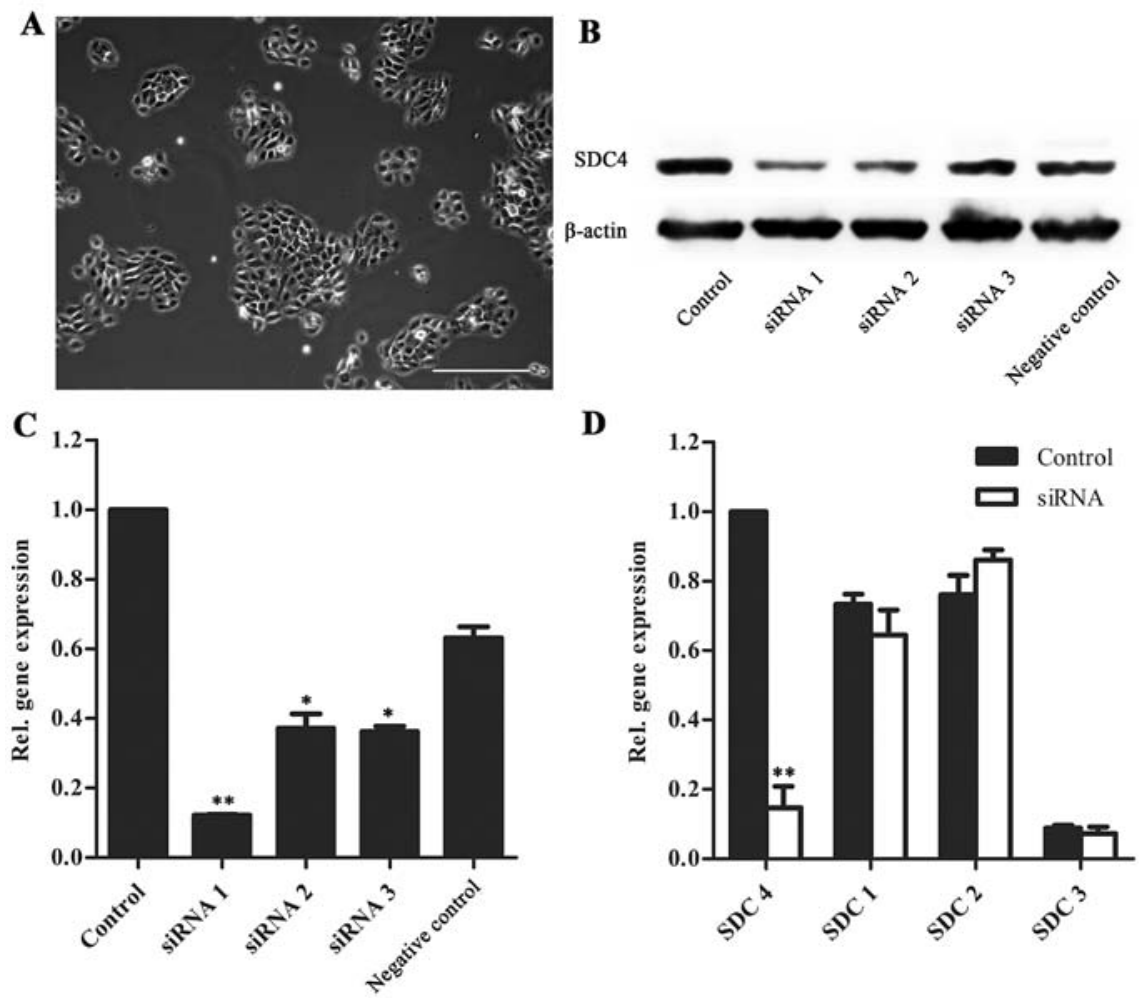

D

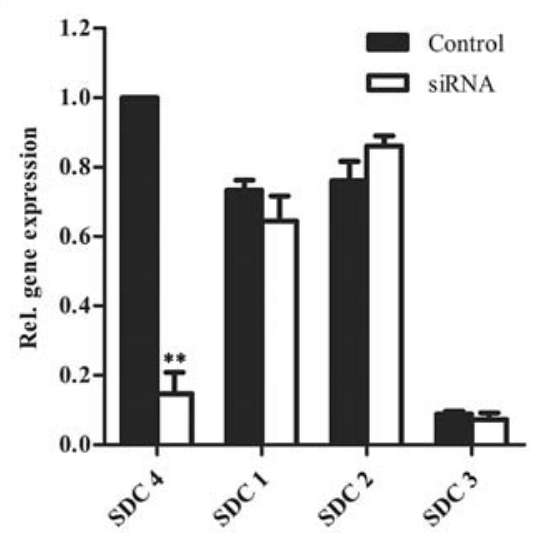

Figure 3. Syndecan-4 (SDC4) silencing in HAT-7 cells. (A) Morphology of HAT-7 cells. Scale bar, $50 \mu \mathrm{m}$. (B and C) RT-qPCR and western blot analysis revealed that SDC4 was successfully knocked down by small interfering RNA (siRNA); siRNA 1 showed the highest inhibition efficiency. (D) siRNA 1 had no effect on the gene expression of SDC1, 2 or 3. All data are presented as the means \pm standard deviation (SD) of triplicate experiments. ${ }^{*} \mathrm{P}<0.05$ and ${ }^{* * *} \mathrm{P}<0.001$.

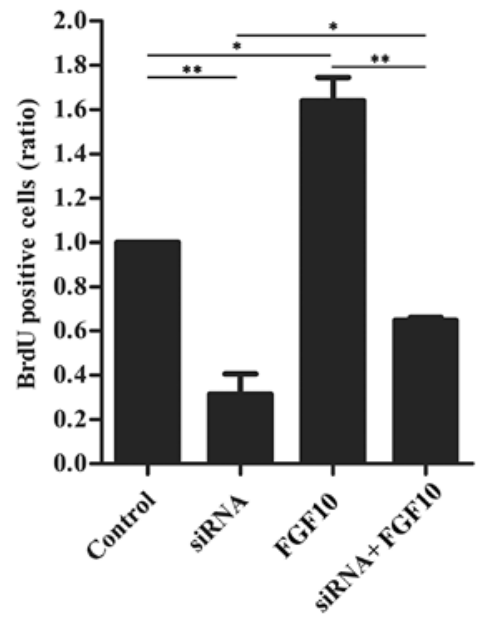

Figure 4. Cell proliferation was detected by BrdU incorporation assay. Cells treated solely with fibroblast growth factor 10 (FGF10) exhibited an approximately 1.6-fold greater number of BrdU-positive cells than the controls, and cells transfected with small interfering RNA (siRNA) a markedly reduced proliferation. FGF10 partially reverse the inhibitory effect exerted by syndecan-4 (SDC4) siRNA. All data are presented as the means \pm standard deviation (SD) of triplicate experiments. ${ }^{*} \mathrm{P}<0.05$ and ${ }^{* *} \mathrm{P}<0.001$.

SDC4 and FGF10 co-regulate HAT-7 cell proliferation. To determine the effects of SDC4 on cell proliferation and the response of these siRNA-treated cells to FGF10, recombinant mouse FGF10 was added to the HAT-7 cell culture medium following transfection, and a BrdU incorporation analysis was then performed (Fig. 4). The cells treated solely with FGF10 exhibited an approximately 1.6-fold greater number of BrdUpositive cells than the controls, and the cells transfected with siRNA exhibited a marked decrease in proliferation. Exogenous FGF10 partially reversed the inhibitory effect exerted by siRNA targeting SDC4.

SDC4 affects HAT-7 cell differentiation and is regulated by FGF10 signaling. Following treatment with siRNA and/or recombinant protein FGF10, the expression of amelogenesisrelated genes were evaluated by RT-qPCR and western blot analysis (Fig. 5). When SDC4 expression was reduced by siRNA, the expression of AMGN, AMBN, KLK4 and matrix metalloproteinase (MMP)20 increased. Enamelin (ENAM) was unaffected and RUNX2 expression slightly decreased when SDC4 expression decreased (Fig. 5A). FGF10 downregulatex the expression of AMGN, AMBN and KLK4. The addition of exogenous FGF10 weakened the effect exerted by siRNA targeting SDC4 and upregulated SDC4 protein expression (Fig. 5B).

\section{Discussion}

Tooth formation is regulated by interactions between the epithelium and the underlying neural crest-derived mesenchyme (15). The earliest morphological sign of tooth formation in mammals is the appearance of the dental lamina, forming as a thickening of oral epithelium. The epithelium of the lamina thickens at sites where teeth will grow, forming dental placodes at these sites. During this bud stage, the dental epithelium segregates into peripheral basal cells (inner and outer enamel epithelium) 

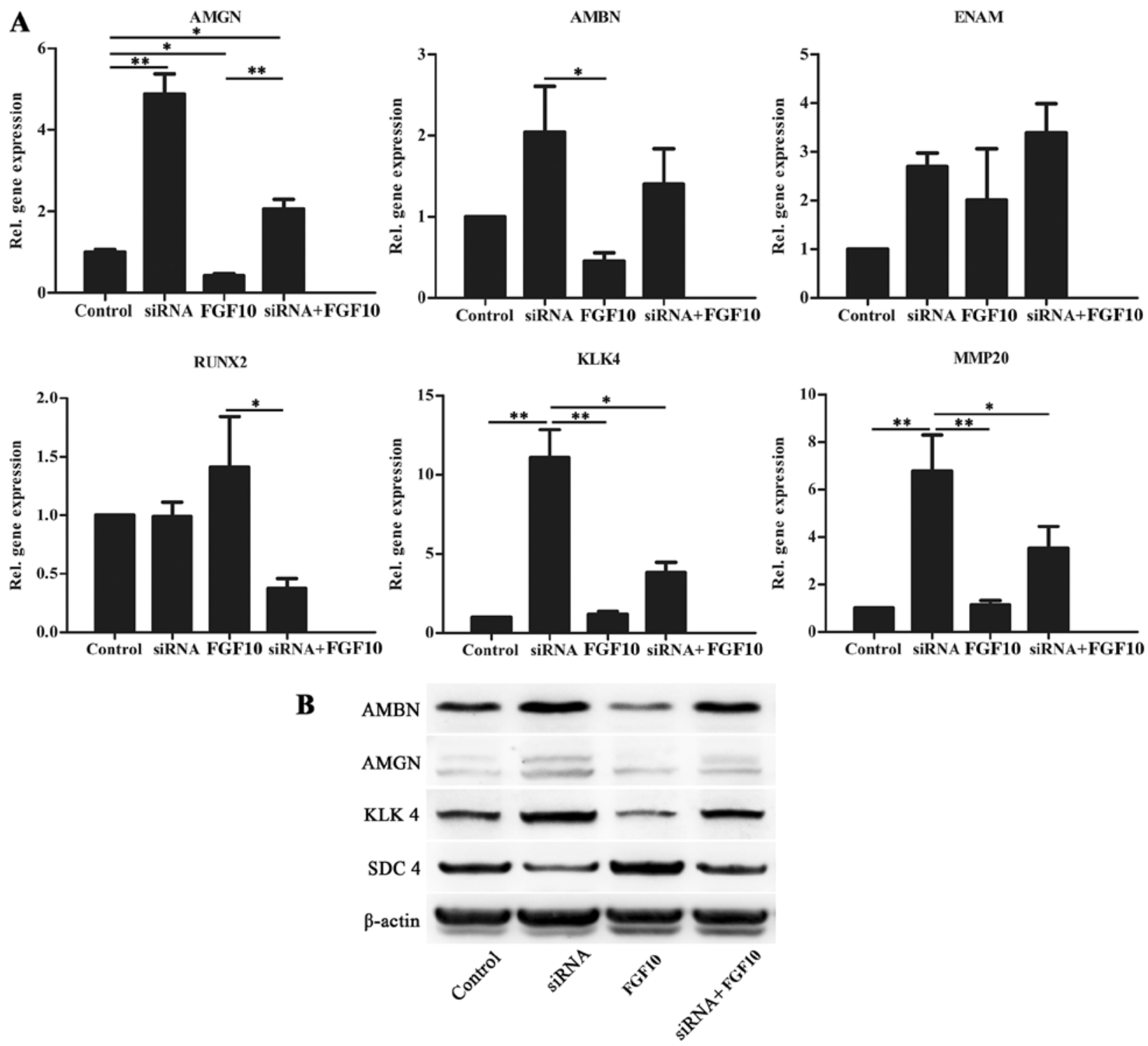

Figure 5. (A and B) Amelogenesis-related gene and protein expression was evaluated by RT-qPCR and western blot analysis. Fibroblast growth factor 10 (FGF10) was downregulated the expression of amelogenin (AMGN), ameloblastin (AMBN), kallikrein 4 (KLK4) and matrix metalloproteinase 20 (MMP20). When SDC4 expression was reduced by small interfering RNA (siRNA), the expression of these 4 genes increased. Enamelin (ENAM) was unaffected and RUNX2 expression slightly decreased when SDC4 expression decreased. FGF10 weakened the effects exerted by siRNA by upregulating SDC4 protein expression. All data are presented as the means \pm standard deviation (SD) of triplicate experiments. ${ }^{*} \mathrm{P}<0.05$ and ${ }^{* * *} \mathrm{P}<0.001$. Rel. gene expression, relative gene expression.

and centrally located loosely arranged cells, termed SR cells. The size and shape of the tooth crown becomes apparent during the cap and bell stages of development. Beginning from late bell stage (E18), the inner enamel epithelium differentiates into pre-ameloblasts and then ameloblasts, which secrete enamel matrix $(16,17)$.

To the best of our knowledge, this study is the first to evaluate SDC4 expression in molar germs at the late bell stage of development. It was detected primarily in the oral epithelium and the dental epithelial cells of the enamel organ in molar germs, including SR cells, the inner and outer enamel epithelium, and pre-ameloblasts, but not in ameloblasts. The intense immunostaining in the SR was decreased along with amelogenesis, possibly due to the retraction of the SR and the decrease in the number of cells $(18,19)$. These results indicate that SDC4 expression patterns may be critically controlled during enamel organ development.
The expression of SDC4 was also analyzed in mouse incisors. Rodent incisors continue to grow throughout adult life due to the presence of dental epithelial stem cells, which give rise to enamel-secreting ameloblasts. Incisors are an excellent model for investigating the molecular underpinnings of amelogenesis as epithelial cells at different stages of differentiation can be easily detected in postnatal incisors. In the present study, SDC4 protein expression was detected in the cervical loops. A loss of SDC4 expression was also evident in the epithelial cells of the postnatal incisors when the inner epithelium gave rise to ameloblasts. In addition, the amount staining in the SI cells gradually decreased along with amelogenesis. Previous studies have demonstrated that epithelial cell behaviors are regulated by an integrated gene regulatory network, among which FGF10 signaling regulates epithelial stem cell proliferation and SI cell differentiation $(12,13)$. FGF10, detected in the mesenchyme surrounding the labial cervical loop, binds to 
FGFR2b, which is expressed in the epithelium of the cervical loop (20). In this study, the distribution of SDC4 in incisors suggests it is closely related to dental epithelial cell differentiation and may be involved in FGF10 signaling.

However, this localization of SDC4 in incisors contradicts earlier findings presented in the study by Muto et al (21), who synthesized rabbit antibody to the ectodomain of the mouse SDC4 core protein and found that SDC4 was expressed throughout the tooth cellular compartments, including ameloblasts. In this study, the anti-SDC4 antibody was the synthetic peptide surrounding the intracellular domain of SDC4 (22). Due to the fact that the cytoplasmic domains of the core protein are conserved, and the extracellular domain of SDC4 can be proteolytically cleaved, a process mediated by a variety of proteases of the MMP family, it can thus be suggested that the SDC4 ectodomain may be cleaved to the ameloblasts that synthesize MMP20 at the secretory stage (23).

SDC4 was also expressed in dental mesenchymal cells adjacent to cervical loops both in molars (E18) and postnatal incisors. In molar germs, the dental mesenchyme is not segregated into the dental papilla and the peripheral dental follicle at the beginning of the bell stage (24). It has recently been suggested that there is a population of putative mesenchymal stem cells between cervical loops in incisors (25). This intriguing similarity between molar and incisor germs suggests that SDC4 may also have a function in regulating dental mesenchymal cells.

Coincidentally, the suggestion of SDC4 functionality was replicated in our in vitro studies. The HAT-7 cells were used here to assess the mechanisms through which SDC4 affects the proliferation and differentiation of dental epithelial cells. The HAT-7 cell line is an immortalized dental epithelial cell line derived from the cervical stem cell population of rat mandibular incisors. It can shift from the transient amplification stage to ameloblast lineage cells (26). SDC4 expression was downregulated by siRNA, which had no effect on the gene expression of SDC1, 2 or 3, the other three known members of the SDC family (27).

Our results revealed that SDC4 expression was associated with low levels of AMGN, AMBN, KLK4 and MMP20, while the specific siRNA knockdown of SDC4 led to a highly significant increase in the expression of these 4 genes; the gene expression of ENAM and RUNX2 was unaffected. As regards the amelogenesis-related genes, AMGN, AMBN and ENAM are scaffold proteins required to support rod formation and hydroxyapatite (HA) crystallization during enamel formation (16). RUNX2 is a key regulatory transcription factor that suppresses genes that are expressed during the secretory stage, such as AMGN and ENAM, and thus regulates enamel formation (28). MMP20 and KLK4 are proteases that break down enamel proteins to form a mineralized layer of enamel (23). Among the genes investigated in this study, they were the most profoundly affected genes when SDC4 expression was inhibited.

In addition, the present study demonstrated that FGF10 promoted cell proliferation and inhibited amelogenesis-related genes expression. Previous studies have demonstrated that FGF10 regulates dental epithelial stem cell proliferation and maintenance. Arrest in FGF10 signaling contributes to the terminal differentiation of the cells (20). Therefore, it is possible that exogenous FGF10 kept the cells in a less differentiated state. Furthermore, the addition of exogenous FGF10 weakened the effects of siRNA through the upregulation of SDC4 protein expression, indicating that SDC4 is under the control of FGF10 signaling.

SDC4 is thought to be involved in the formation of the receptor-ligand complex $(29,30)$. It is possible that the ternary combination of FGF10, its receptor and SDC4 decrease when SDC4 expression decreases. In this study, we demonstrate that SDC4 may regulate epithelial cell behaviors through FGF10 signaling. However, several heparin-binding growth factors interact with SDC4 and may also regulate cell proliferation and differentiation during tooth development (31). The specific mechanisms through which SDC4 affects amelogenesis remain to be elucidated in future sutdies.

\section{Acknowledgements}

This study was supported by the National Basic Research Program (China, 2010CB944800), the National High-Technology Research and Development Program (China, 2011AA030107), the Nature Science Foundation of China (China, 81271095, 81271119 and 81200792), the International Cooperation Program of China (China, 2013DFG32770 and 2011DFA51970), the China Postdoctoral Science Foundation (China, 2012M511934), the Key Technology R\&D Program of Sichuan Province (2012SZ0013, 12ZC0493, 13ZC0971, 2013GZX0158 and 13ZC0979) and the Basic Research Program of Sichuan Province (12JC0212 and 2013JY0019).

\section{References}

1. Choi Y, Chung H, Jung H, Couchman JR and Oh ES: Syndecans as cell surface receptors: Unique structure equates with functional diversity. Matrix Biol 30: 93-99, 2011.

2. Bellin RM, Kubicek JD, Frigault MJ, et al: Defining the role of syndecan-4 in mechanotransduction using surface-modification approaches. Proc Natl Acad Sci USA 106: 22102-22107, 2009.

3. Elfenbein A and Simons M: Syndecan-4 signaling at a glance. J Cell Sci 126: 3799-3804, 2013.

4. Tkachenko E, Rhodes JM and Simons M: Syndecans: new kids on the signaling block. Circ Res 96: 488-500, 2005.

5. Kuriyama S and Mayor R: A role for Syndecan-4 in neural induction involving ERK- and PKC-dependent pathways. Development 136: 575-584, 2009.

6. Iwabuchi T and Goetinck PF: Syndecan-4 dependent FGF stimulation of mouse vibrissae growth. Mech Dev 123: 831-841, 2006.

7. Shimokawa K, Kimura-Yoshida C, Nagai N, et al: Cell surface heparan sulfate chains regulate local reception of FGF signaling in the mouse embryo. Dev Cell 21: 257-272, 2011.

8. Goodwin AF, Tidyman WE, Jheon AH, et al: Abnormal Ras signaling in Costello syndrome (CS) negatively regulates enamel formation. Hum Mol Genet 23: 682-692, 2014.

9. Huang Z, Kim J, Lacruz RS, et al: Epithelial-specific knockout of the Rac1 gene leads to enamel defects. Eur J Oral Sci 119 (Suppl 1): S168-S176, 2011.

10. Porntaveetus T, Otsuka-Tanaka Y, Basson MA, Moon AM, Sharpe PT and Ohazama A: Expression of fibroblast growth factors (Fgfs) in murine tooth development. J Anat 218: 534-543, 2011.

11. Kettunen P and Thesleff I: Expression and function of FGFs-4, -8 , and -9 suggest functional redundancy and repetitive use as epithelial signals during tooth morphogenesis. Dev Dyn 211: 256-268, 1998

12. Harada H, Toyono T, Toyoshima K, et al: FGF10 maintains stem cell compartment in developing mouse incisors. Development 129: 1533-1541, 2002. 
13. Wang XP, Suomalainen M, Felszeghy S, et al: An integrated gene regulatory network controls stem cell proliferation in teeth. PLoS Biol 5: e159, 2007.

14. Kawano S, Morotomi T, Toyono T, Nakamura N, Uchida T, Ohishi M, Toyoshima K and Harada H: Establishment of dental epithelial cell line (HAT-7) and the cell differentiation dependent on Notch signaling pathway. Connect Tissue Res 43: 402-412, 2002.

15. Blanpain C,Horsley V and Fuchs E: Epithelial stem cells: turning over new leaves. Cell 128: 445-458, 2007.

16. Hu JC, Sun X, Zhang C and Simmer JP: A comparison of enamelin and amelogenin expression in developing mouse molars. Eur J Oral Sci 109: 125-132, 2001.

17. Thesleff I and Tummers M: Tooth organogenesis and regeneration. In: StemBook. Harvard Stem Cell Institute, Cambridge, MA, 2008

18. Ida-Yonemochi H, Satokata I, Ohshima H, et al: Morphogenetic roles of perlecan in the tooth enamel organ: an analysis of overexpression using transgenic mice. Matrix Biol 30: 379-388, 2011

19. Baratella L, Arana-Chavez VE and Katchburian E: Apoptosis in the early involuting stellate reticulum of rat molar tooth germs. Anat Embryol (Berl) 200: 49-54, 1999.

20. Harada H, Kettunen P, Jung HS, Mustonen T, Wang YA and Thesleff I: Localization of putative stem cells in dental epithelium and their association with notch and FGF signaling. J Cell Biol 147: 105-120, 1999.

21. Muto T, Miyoshi K, Munesue S, et al: Differential expression of syndecan isoforms during mouse incisor amelogenesis. J Med Invest 54: 331-339, 2007.

22. Corti F, Finetti F, Ziche M and Simons M: The syndecan-4/protein kinase $\mathrm{C} \alpha$ pathway mediates prostaglandin E2-induced extracellular regulated kinase (ERK) activation in endothelial cells and angiogenesis in vivo. J Biol Chem 288: 12712-12721, 2013.
23. Bartlett JD, Yamakoshi Y, Simmer JP, Nanci A and Smith CE: MMP20 cleaves E-cadherin and influences ameloblast development. Cells Tissues Organs 194: 222-226, 2011.

24. Rothová M, Peterková R and Tucker AS: Fate map of the dental mesenchyme: dynamic development of the dental papilla and follicle. Dev Biol 366: 244-254, 2012.

25. Seidel K, Ahn CP, Lyons D, et al: Hedgehog signaling regulates the generation of ameloblast progenitors in the continuously growing mouse incisor. Development 137: 3753-3761, 2010.

26. Matsumoto $A$, Harada $H$, Saito $M$ and Taniguchi A: Induction of enamel matrix protein expression in an ameloblast cell line co-cultured with a mesenchymal cell line in vitro. In Vitro Cell Dev Biol Anim 47: 39-44, 2011.

27. Xian X, Gopal S and Couchman JR: Syndecans as receptors and organizers of the extracellular matrix. Cell Tissue Res 339: 31-46, 2010.

28. Athanassiou-Papaefthymiou M, Kim D, Harbron L, et al: Molecular and circadian controls of ameloblasts. Eur J Oral Sci 119 (Suppl 1): S35-S40, 2011

29. Elfenbein A, Lanahan A, Zhou TX, et al: Syndecan 4 regulates FGFR1 signaling in endothelial cells by directing macropinocytosis. Sci Signal 5: ra36, 2012.

30. Horowitz A, Tkachenko E and Simons M: Fibroblast growth factor-specific modulation of cellular response by syndecan- 4 . J Cell Biol 157: 715-725, 2002.

31. Thesleff I, Vaahtokari A and Partanen AM: Regulation of organogenesis. Common molecular mechanisms regulating the development of teeth and other organs. Int J Dev Biol 39: 35-50, 1995. 